\section{LONG-TERM CARDIAC AND VASCULAR PHENOTYPE OF YOUNG WOMEN WITH PREGNANCIES COMPLICATED BY PREECLAMPSIA}

doi:10.1136/heartjnl-2012-301877b.143

${ }^{1} \mathrm{M}$ Lazdam, ${ }^{*} \mathrm{~A}$ de la Horra, ${ }^{1} \mathrm{~J}$ Diesch, ${ }^{1} \mathrm{~J}$ Francis, ${ }^{1} \mathrm{Y}$ Kenworthy, ${ }^{2} \mathrm{~A}$ Shore, ${ }^{1} \mathrm{C}$ Redman, ${ }^{1} \mathrm{~S}$ Neubauer, ${ }^{1} \mathrm{R}$ Kharbanda, ${ }^{1} \mathrm{~N}$ Alp, ${ }^{1} \mathrm{~B}$ Kelly, ${ }^{1} \mathrm{P}$ Leeson. ${ }^{1}$ University of Oxford, Oxford, UK; ${ }^{2}$ Peninsula College of Medicine and Dentistry, Exeter, UK

Background Preeclampsia is an independent risk factor for cardiovascular disease and is associated with a range of maternal cardiovascular abnormalities during the perinatal period. We studied mothers late after preeclampsia to define persistent cardiac and vascular changes that might link preeclampsia to later disease and identified key pregnancy factors that predicted the variation.

Methods 140 women aged 28-50 years were studied 6-13 years after the index pregnancy. 90 had preeclampsia (45 early onset before 34 weeks gestation and 45 later onset) and 50 had normotensive uncomplicated pregnancies. Women with cardiovascular risk factors before pregnancy were excluded. Central blood pressure (BP) and arterial stiffness (pulse wave velocity (PWV)/augmentation index (AI)) were assessed by applanation tonometry, common carotid intima media thickness (cIMT) by ultrasound, cutaneous capillary density by intravital microscopy and endothelial function by flow mediated dilatation (FMD). 46 women returned for assessment of cardiac structure and function by magnetic resonance and echocardiography as well as ambulatory blood pressure monitoring. Fasting lipids, glucose, insulin and circulating cytokines and adhesion molecules were measured in all subjects.

Results Women with a previous history of preeclampsia had $4-12 \mathrm{~mm} \mathrm{Hg}$ higher peripheral and central BP $(p<0.001)$ as well as characteristic differences in ambulatory measures. They also had increased arterial stiffness (ANOVA $p=0.04$ ), cIMT (ANOVA $p=0.006$ ) and capillary rarefaction (ANOVA $p=0.005$ ). Cardiac size and systolic function were preserved but there was evidence of abnormal diastolic relaxation (E/E' - ANOVA $\mathrm{p}=0.04)$ and elevated total: HDL cholesterol $(p=0.003)$, insulin resistance $(p=0.04)$, circulating TNF $\alpha(p=0.007)$ and eSelectin $(p<0.001)$. All changes were graded according to the timing and severity of preeclampsia. Conclusion Structural micro and macrovascular changes predominate in young women a decade after preeclampsia. Alterations in metabolic markers and mild changes in diastology are also evident. Timing and severity of preeclampsia are predictive of these differences and may identify women at greatest potential benefit from primary prevention advice.

\section{NURSES MEET THE CHALLENGE OF HELPING HIGH CVD RISK SMOKERS TO QUIT WITH THE HELP OF VARENICLINE IN A PREVENTIVE CARDIOLOGY PROGRAMIME}

doi:10.1136/heartjnl-2012-301877b.144

${ }^{1} \mathrm{C} S$ Jennings, ${ }^{*}{ }^{1} \mathrm{~K}$ Kotseva, ${ }^{2} \mathrm{D}$ De Bacquer, ${ }^{3} \mathrm{~A}$ Hoes, ${ }^{4} \mathrm{~J}$ De Velasco, ${ }^{5} \mathrm{~S}$ Brusaferro, ${ }^{6} \mathrm{~S}$ Tonstad, ${ }^{1} \mathrm{D}$ A Wood. ${ }^{1}$ Imperial College London, London, UK; ${ }^{2}$ Ghent University, Ghent, Belgium; ${ }^{3}$ University Medical Center Utrecht-Julius Centre for Health Sciences and Primary Care, Utrecht, Netherlands; ${ }^{4}$ Department of Cardiology, University General Hospital of Valencia, Valencia, Spain, ${ }^{5}$ University Hospital, Udine, Italy; ${ }^{6}$ Oslo University Hospital, Oslo, Norway

Background The EUROACTION plus study aimed to assess effectiveness of a nurse-led preventive cardiology programme for high CVD risk smokers willing to make a quit smoking attempt compared to usual care in general practice.

Methods High CVD risk smokers aged to $18-80$ years with vascular disease and 50-80 years at high risk of developing CVD (Heartscore $\geq 5 \%$ over 10 years, or treated for risk factors or DM) and their partners were individually randomised to either the programme $(\mathrm{EA}+)$ or usual care (UC). EA+ patients had optional access to free varenicline and met with the study nurse every 2 weeks to support their quit attempt and to have dietary and physical activity advice and CVD risk factor management. The primary outcome was self reported 7-day point prevalence of abstinence validated with breath carbon monoxide of $<10 \mathrm{ppm}$. Analysis was by intention to treat (ITT). Follow-up was at 16 weeks.

Results 696 patients were recruited: 350 randomised to EA+ and 346 to UC. $85 \%$ EA+ and $83 \%$ UC returned at 16 weeks. $91 \%$ of patients in EA+ chose to use varenicline to help them to quit smoking and $51 \%$ of $E A+$ patients quit compared to $19 \%$ in UC (OR $4.5295 \%$ CI 3.2 to $6.4 p<0.0001)$. In those who fully participated in $\mathrm{EA}+63 \%$ quit smoking compared to $17 \%$ who did not complete and $22 \%$ who did not participate. At follow-up self reported health related quality of life (HRQoL) was better in EA+ with significantly higher mean EQ-VAS scores in EA+ (74/100) compared to UC (70, $\mathrm{p}=0.002)$. Functional limitation profile scores (SF36) improved in EA + during the programme $(25.6-26.2 \Delta+0.5695 \%$ CI 0.23 to 0.88 $\mathrm{p}=0.0009$ ). No differences were seen in depression scores (HADS), but anxiety scores reduced in EA+ during the programme (5.63-5.27 $\Delta-0.3595 \% \mathrm{CI}-0.67$ to $-0.03 \mathrm{p}=0.03)$.

Conclusion Intensive support from nurses with optional use of varenicline was successful in helping over half of all high CVD risk smokers to quit. This was associated with a reduction in anxiety and increased quality of life.

\section{EUROACTION PLUS: A RANDOMISED CONTROLLED TRIAL ON PREVENTIVE CARDIOLOGY PROGRAMME PLUS INTENSIVE SMOKING CESSATION WITH VARENICLINE FOR VASCULAR AND HIGH CVD RISK SMOKERS AND THEIR PARTNERS - PRINCIPAL RESULTS}

doi:10.1136/heartjnl-2012-301877b.145

${ }^{1} \mathrm{~K}$ Kotseva, ${ }^{*} \mathrm{C}$ Jennings, ${ }^{2} \mathrm{D}$ De Bacquer, ${ }^{3} \mathrm{~A}$ Hoes, ${ }^{4} \mathrm{~J}$ De Velasco, ${ }^{5} \mathrm{~S}$ Brusaferro, ${ }^{1} \mathrm{~J}$ Jones, ${ }^{1} \mathrm{~A}$ Mead, ${ }^{6} \mathrm{~S}$ Tonstad, ${ }^{1} \mathrm{D}$ Wood. ${ }^{1}$ National Heart \& Lung Institute, Imperial College London, London, UK; ${ }^{2}$ Ghent University, Ghent, Belgium; ${ }^{3}$ University Medical Center Utrecht, Utrecht, Netherlands; ${ }^{4}$ University General Hospital of Valencia, Valencia, Spain; ${ }^{5}$ University Hospital "Santa Maria della Misericordia", Udine, Italy; ${ }^{6}$ Oslo University Hospital, Oslo, Norway

Aim The aim of the EUROACTION PLUS trial was to determine if the nurse-led preventive cardiology programme in primary care, with an intensive smoking intervention including the optional use of Varenicline, could achieve more effective smoking abstinence among persistent smokers with either established vascular disease, or at high risk of developing cardiovascular disease and to reduce overall cardiovascular risk compared to usual care (UC).

Methods EUROACTION PLUS (EA PLUS) was a randomised controlled intervention trial carried out in general practices across 4 European countries: Italy, The Netherlands, Spain and the UK. Vascular patients and people at high risk of developing cardiovascular disease who were current smokers were individually randomised to receive either a professional smoking cessation intervention, which included the optional use of Varenicline, delivered in the context of the nurse-led EUROACTION preventive cardiology programme, or their usual care. The primary outcome was the proportion of non-smokers (7-day prevalence of nonsmoking) validated by breath $\mathrm{CO}(<10 \mathrm{ppm})$ in intervention compared to usual care at 16 weeks. The secondary outcomes included the proportions of patients achieving the Joint European Societies lifestyle, risk factor and therapeutic targets for cardiovascular disease prevention.

Results 696 patients were recruited: 350 randomised to EA PLUS and 346 to UC. $85 \%$ EA PLUS and $83 \%$ UC returned at 16 weeks. For 